MITP/21-002, MSUHEP-20-016

\title{
MultivariateApart: Generalized Partial Fractions
}

\author{
Matthias Heller ${ }^{\mathrm{a}}$, Andreas von Manteuffel ${ }^{\mathrm{b}}$ \\ ${ }^{a}$ PRISMA ${ }^{+}$Cluster of Excellence, Johannes-Gutenberg Universität, \\ 55099 Mainz, Deutschland \\ ${ }^{b}$ Department of Physics and Astronomy, Michigan State University, \\ East Lansing, Michigan 48824, USA
}

\begin{abstract}
We present a package to perform partial fraction decompositions of multivariate rational functions. The algorithm allows to systematically avoid spurious denominator factors and is capable of producing unique results also when being applied to terms of a sum separately. The package is designed to work in Mathematica, but also provides interfaces to the Form and Singular computer algebra systems.
\end{abstract}

Email addresses: maheller@students.uni-mainz.de (Matthias Heller), vmante@msu.edu (Andreas von Manteuffel) 


\section{Contents}

1 Introduction 2

2 Motivation 3

2.1 Spurious poles in iterated partial fractions . . . . . . . . . . 3

2.2 Features of Leunartas' decomposition . . . . . . . . . . . . . . 4

2.3 A wish-list for a "good" partial fractioning algorithm . . . . . . . 6

3 Multivariate partial fractions with polynomial reduction 7

3.1 Reduction algorithm . . . . . . . . . . . . . . . . 7

3.2 Monomial ordering . . . . . . . . . . . . . . . . . . 8

3.3 Example for the algorithm . . . . . . . . . . . . . . . . . 9

3.4 Efficient reduction of factorized inputs . . . . . . . . . . . 11

3.5 A comment on rational reconstructions . . . . . . . . . . . . 11

4 The package 13

4.1 Installation and basic usage . . . . . . . . . . . . . . . 13

4.2 Advanced usage pattern . . . . . . . . . . . . . . . . . . 14

4.3 Interface with Singular . . . . . . . . . . . . . . . . . . 16

4.4 Interface with Form . . . . . . . . . . . . . . . . . . 17

5 Applications $\quad \mathbf{1 8}$

5.1 One-loop amplitudes for $\gamma^{*} \gamma^{*} \rightarrow l^{+} l^{-}$with finite lepton mass . . 18

5.2 Two-loop amplitudes for $g g \rightarrow Z Z$ with a closed top-quark loop . 19

5.3 Two-loop amplitudes for massless five-particle scattering . . . . . 20

6 Conclusion and outlook 2

\section{Appendices}

A Polynomial reductions 23

B Leĭnartas' requirements and polynomial reductions 25

\section{Introduction}

Employing partial fraction decompositions to bring analytic expressions into a unique form has a long history in particle physics. Already one of the first computer algebra systems (CAS), Veltman's program Schoonschip [1], that was developed in 1963 to calculate radiative corrections, had a function to partial fraction products of two or three rational functions [2]. With Schoonship's successor Form [3, 4], partial fraction decompositions of rational functions became widely established in the particle physics community. While the standard partial fraction decomposition is a method for rational functions of a single variable, it is often applied iteratively to treat multivariate functions. However, this generalization has two drawbacks: first this method generally introduces new 
denominator factors, i.e. spurious singularities, and second, it is often slower than reduction schemes in which spurious singularities are avoided from the beginning. An alternative representation which avoids spurious denominators is known as Leĭnartas' decomposition [ [5, 6, 7, 8, 8]. It separates denominators which don't share common zeros or are algebraically dependent.

Here, we discuss a method to compute a partial fraction decomposition via polynomial reductions. Our general approach coincides with that of Ref. [9], but we present new insights concerning the choice of a good monomial ordering and the resulting output forms. In particular, we propose a new monomial ordering, which still guarantees the separation of denominator zeros but allows for deviations from Leĭnartas' form to allow for lower denominator degrees. We also discuss performance optimizations and other practical issues, such as the local elimination of specific denominators, if they are known to be spurious globally. We implement our algorithms in a Mathematica package which is publicly available. It can be used to partial fraction rational functions directly in Mathematica, but also allows to generate local replacement rules that can be incorporated in Form.

The outline of the paper is as follows. In Section 2 we motivate our method. We demonstrate the problem with iterated univariate partial fractions and discuss ambiguities when applying the "classical" Leinnartas decomposition to individual terms of a sum. We spell out an explicit wish-list for an ideal partial fraction algorithm and discuss which items are addressed by available implementations and the one described in this article, respectively. In Section 3 we introduce our algorithms in detail, give examples and show how it can be used efficiently in complicated cases. Furthermore, we present some considerations concerning the reconstruction of rational functions from finite field samples, which can be useful to speed-up tasks like linear system solving. In Section 4 we give an introduction to our Mathematica package. We describe the relevant functions and give examples for their usage. In Section 5 we list "real world" applications of our methods to the calculation of Feynman amplitudes, often resulting in significantly reduced sizes of the mathematical expressions. In Section [6] we conclude and provide an outlook. Appendix $\mathrm{A}$ gives a brief introduction to polynomial reductions. Appendix B discusses the relation between Leinnartas' decomposition and our polynomial reduction method.

\section{Motivation}

\subsection{Spurious poles in iterated partial fractions}

We consider a univariate rational function $r(x)=n(x) / d(x)$, where $n$ and $d$ are polynomials in $x$ and the coefficient field $K$ could be e.g. the set of rational numbers. The denominator $d(x)$ can be factored into irreducible factors $d_{i}(x)$ such that $d(x)=\prod_{i} d_{i}^{\alpha_{i}}(x)$, where the polynomials $d_{i}$ can not be written as a product of two non-constant polynomials and $\alpha_{i} \in \mathbb{N}$. The univariate partial 
fraction decomposition of $r(x)$ is given by

$$
r(x)=\sum_{i} \sum_{j \leq \alpha_{i}} \frac{n_{i}(x)}{d_{i}^{j}(x)}
$$

where in each term the degree of the numerator is smaller than the degree of the denominator. As an example, consider the partial fraction decomposition

$$
\frac{x}{(x-1)(x+1)^{2}}=-\frac{1}{4(x+1)}+\frac{1}{2(x+1)^{2}}+\frac{1}{4(x-1)} .
$$

In the case of multivariate rational functions $r(x, y, \ldots)$ the partial fractioning can be performed iteratively in each variable. One performs a partial fraction decomposition with respect to the first variable $x$, treating the other variables as constants during this step. The resulting coefficients $p_{i}$ are now rational functions of the remaining variables, and one can perform a partial fraction decomposition of the $p_{i}(y, \ldots)$ with respect to the next variable $y$. One iterates until the coefficients are actual numbers. As an example, consider the function $r(x, y)=1 /((x-f(y))(x-g(y)))$, where $f(y)$ and $g(y)$ are two different polynomials of $y$. A partial fractioning with respect to $x$ gives

$$
\frac{1}{(x-f(y))(x-g(y))}=\frac{1}{(f(y)-g(y))} \frac{1}{(x-f(y))}-\frac{1}{(f(y)-g(y))} \frac{1}{(x-g(y))} .
$$

The partial fractioning in $x$ thus introduces a denominator $f(y)-g(y)$, which may have zeros at regular points of the original rational function $r(x, y)$. For example, consider the special case

$$
\frac{1}{(x+y)(x-y)}=\frac{1}{2 y} \frac{1}{(x-y)}-\frac{1}{2 y} \frac{1}{(x+y)},
$$

where the subsequent partial fractioning of the $x$-independent coefficients in $y$ is trivial. Although the original expression is manifestly regular at $y=0$, the individual terms of the partial fractioned form are not - they introduce spurious poles $1 / y$. This obscures the interpretation of the singularity structure of the expression and can lead to loss of precision in its numerical evaluation close to $y=0$. Note that a different spurious pole would have appeared in this example if we had chosen to first partial fraction in $y$ and then in $x$.

We conclude that iterated partial fractioning will in general introduce spurious poles and is therefore not an ideal approach to the multivariate case.

\subsection{Features of Leĭnartas' decomposition}

Leinnartas' decomposition is an approach to multivariate partial fractioning, which avoids the introduction of spurious singularities. In this Section, we review this decomposition and comment on possible ambiguities arising in practical calculations. Polynomial reductions and the usage of Gröbner bases will play an important role here and in the following; in case the reader is not familiar with these concepts we recommend to read Appendix $\mathrm{A}$ first. 
Definition 1. Leinartas' decomposition [5, 6] of a rational function $r$ of the variables $x_{1}, \ldots, x_{n}$ with coefficients in the field $K$ is a decomposition of the form

$$
r\left(x_{1}, \ldots\right)=\sum_{\mathcal{S}} \frac{n_{\mathcal{S}}\left(x_{1}, \ldots\right)}{\prod_{i \in \mathcal{S}} d_{i}^{\alpha_{i}}\left(x_{1}, \ldots\right)},
$$

where, for each term of the decomposition, $\mathcal{S}$ is an index set, such that all denominators $d_{i}$ with $i \in \mathcal{S}$

(i) have common zeros in $\bar{K}^{n}$, and

(ii) are algebraically independent.

Here, $\bar{K}$ denotes the algebraic closure of $K$, e.g. the algebraic numbers for $K=\mathbb{Q}$ or $\mathbb{C}$ for $K=\mathbb{R}$.

A set of polynomials $\left\{d_{1}, \ldots, d_{m}\right\}$ is called algebraically dependent if there exists a non-zero polynomial $A$ in $m$ variables such that $A\left(d_{1}, \ldots, d_{m}\right)=0$, see e.g. [10, 11]. $A$ is called the annihilator of the ideal generated by the polynomials $\left\{d_{1}, \ldots, d_{m}\right\}$. The annihilator can be obtained by calculating the Gröbner basis of the ideal $\left\{y_{1}-d_{1}\left(x_{1}, \ldots, x_{n}\right), \ldots, y_{m}-d_{m}\left(x_{1}, \ldots, x_{n}\right)\right\}$, if one chooses a monomial ordering in which $y_{i} \prec x_{j} \forall i, j$. A set of polynomials is called algebraically independent if it is not algebraically dependent. Since for $n$ variables at most $n$ polynomials are algebraically independent, there can be at most $n$ different denominator factors for each term in (5).

Algorithm 1. Lěnnartas' decomposition of a rational function can be reached in two reduction steps [6]:

1. Use Hilbert's Nullstellensatz to decompose the denominator of $r$ into several terms such that each term fulfills (i).

2. Calculate the annihilator for each term and use it to decompose each denominator to reach (ii).

In contrast to iterated partial fractioning, these decomposition steps do not introduce new singularities. Appendix B gives more details for this algorithm. A decomposition that fulfills requirements (i) and (ii) is not unique. In order to resolve this ambiguity, Refs. [8, 12] require additionally for each term (5), that the numerator $p_{\mathcal{S}}$ is reduced with respect to the ideal generated by the denominators $\left\{d_{i} \mid i \in \mathcal{S}\right\}$. We note that the algorithm of [12] assumes the input expression to be a single rational function $r=n / d$ with polynomials $n$ and $d$ and starts with a factorization of $d$.

Further ambiguities can arise due to spurious denominators which are not automatically removed. The algorithm in [12] for example does not guarantee a unique result, if $n$ and $d$ are not coprime. However, common factors between numerator and denominator can always be eliminated by first performing a greatest-common-divisor computation.

In practical applications one also encounters sums of rational functions, and it can be useful to decompose individual terms of the sum separately instead of 
combining them over a common denominator first. With the methods spelled out so far, uniqueness is not guaranteed in this approach, even in absence of any spurious denominator. Let us illustrate this phenomenon by considering an example where

$$
r(x, y)=\frac{2 x-y}{x(x+y)(x-y)} .
$$

Then a Lelnartas decomposition of $r$ is given by

$$
r(x, y)=\frac{1}{x(x+y)}+\frac{1}{(x-y)(x+y)} .
$$

Another Leĭnartas decomposition is given by

$$
r(x, y)=\frac{3}{2 x(x+y)}+\frac{1}{2 x(x-y)} .
$$

If we consider the terms in Eqs. (77) and (8) separately, each of them is in Lelnartas'decomposed form and the numerator is reduced with respect to the denominator, but the resulting representation is different for the respective sums. In order resolve this ambiguity, some kind of "global" information needs to be incorporated into the decomposition of "local" terms.

\subsection{A wish-list for a "good" partial fractioning algorithm}

Lets assume someone gives us a rational expression in any form, expanded, over a common denominator, or partially mixed. Then an "ideal" partial fraction decomposition would have the following properties

(i) it should give a unique answer,

(ii) it should not introduce spurious denominator factors,

(iii) it should commute with summation,

(iv) it should eliminate spurious denominators if they are present in the input.

Note that requirement (iii) can be crucial, if one aims at employing such a partial fraction algorithm in a system like Form. In such a case one usually starts with fully expanded expressions, uses local replacement rules, and then wants to obtain a unique answer, such that cancellations can take place.

Unfortunately, there is no know solution to fulfil all of these points simultaneously. In the next Section, we consider an algorithm that fulfills requirements (i), (ii) and (iii), and, in cases in which one knows which denominators are spurious in the final answer, also point (iv). 


\section{Multivariate partial fractions with polynomial reduction}

\subsection{Reduction algorithm}

Let $\left\{d_{1}, \ldots d_{m}\right\}$ be the irreducible denominators of a rational function or a sum of rational functions with $d_{i} \in K\left[x_{1}, \ldots, x_{n}\right]$. Consider the ideal

$$
I=\left\langle q_{1} d_{1}\left(x_{1}, \ldots\right)-1, \ldots, q_{m} d_{m}\left(x_{1}, \ldots\right)-1\right\rangle,
$$

where $I \subset K\left[q_{1}, \ldots, q_{m}, x_{1}, \ldots, x_{n}\right]$ and $q_{i}$ label inverse denominators. The main idea here is that setting the generators of the ideal $q_{i} d_{i}\left(x_{1}, \ldots\right)-1$ to zero corresponds to the relation $q_{i}=1 / d_{i}\left(x_{1}, \ldots\right)$. If we rewrite a rational function $r$ as a polynomial in the variables $q_{1}, \ldots, x_{1}, \ldots$ and reduce it with respect to the ideal $I$, we do not introduce new denominator factors and obtain a unique representation. Furthermore, whenever we encounter a product $q_{i} d_{i}$ it will be reduced to 1 . By choosing a suitable monomial ordering, one can control further features of the reduced form as will be discussed in more detail in Section 3.2 and Appendix B Here, we only note that for any monomial ordering, which sorts first for the $q_{i}$ and then for the $x_{i}$, denominators with disjoint zeros will be separated and it is justified to call the reduction a partial fraction decomposition. Using such a monomial ordering, we thus achieve a multivariate partial fraction decomposition with the following two steps: 1 . calculate the Gröbner basis of the ideal $I$ and 2. reduce the rational function with respect to this Gröbner basis. This reduction yields a unique remainder, which is the partial fractioned form of $r$. A complete algorithm to bring a rational function into a unique partial fractioned form can be formulated as follows (see also [9]).

Algorithm 2. Multivariate partial fraction decomposition. A rational function $r\left(x_{1}, \ldots\right) \in K\left[x_{1}, \ldots\right]$ can be decomposed into partial fractions using the following steps.

1. Bring the rational function into the form $n\left(x_{1}, \ldots\right) / d\left(x_{1}, \ldots\right)$ and cancel common factors in $n$ and $d$ such that they are coprime.

2. Factorize $d$ over $K$. Let's call the irreducible factors of the denominator $d_{i}\left(x_{1}, \ldots\right)$ for $i=1, \ldots, m$.

3. For each denominator factor $d_{i}\left(x_{1}, \ldots\right)$ introduce a new indeterminant $q_{i}$ which represents the inverse of $d_{i}\left(x_{1}, \ldots\right)$, i.e. $q_{i}=1 / d_{i}\left(x_{1}, \ldots\right)$. Express all denominators in the problem in terms of the $q_{i}$ such that the rational function becomes a polynomial $p \in K\left[q_{1}, \ldots, x_{1}, \ldots\right]$.

4. Calculate the Gröbner basis of the ideal $I$ generated by $\left\{q_{1} d_{1}\left(x_{i}\right)-1, \ldots\right.$, $\left.q_{m} d_{m}\left(x_{i}\right)-1\right\}$ using a monomial ordering which sorts first for the $q_{i}$ and then for the $x_{i}$.

5. Find the fully reduced form of the polynomial $p$ with respect to the Gröbner basis.

6. Replace back $q_{i} \rightarrow 1 / d_{i}$.

Step 1 eliminates spurious poles and ensures a unique final form. When operating on large sums, it may be useful to skip this step and operate on the 
invidual terms of the sum separately. In this situation, one can still arrive at a unique output for the sum when considering the decomposition of each term separately, but using the global set of denominators in the sum. For instance, for the example of Section 2.2. Algorithm2 finds a unique form independent of the specific initial form of $r$ (Eqs. (7) and (8)) once all possible denominators in the problem are specified. The difference with respect to the standard Leinartas decomposition lies in the fact, that we specify by the monomial ordering which denominators are preferred in the partial fractioned result. The elimination of denominators which are known to be spurious can then be achieved by a suitable choice of the monomial ordering; this will be discussed in the next Section.

Step 6 is optional, of course. Leaving the denominators in abbreviated form can allow for more compact representations and as a preparation for an efficient numerical implementation.

\subsection{Monomial ordering}

The choice of monomial ordering has a crucial impact on the properties of the output of Algorithm 2 and the performance of its computation. We show in Appendix B that the output form will satisfy Leinartas' requirement (i) and thus separate denominators with disjoint zeros as long as the monomial ordering orders all $q_{i}$ before the $x_{i}$, while Leunartas' requirement (ii) may or may not be satisfied depending on further details.

The calculation of the Gröbner basis can be very challenging for practical applications of Algorithm 2, depending heavily on the choice of monomial ordering. Here we propose a specific monomial ordering which aims to provide good computational performance. We tested this ordering in various calculations of scattering amplitudes, and we were able to compute the Gröbner basis and partial fraction the required rational functions in all cases that we considered.

To motivate our choice of monomial ordering, let us go back to Eq. (3). Note that the prefactors in front of the denominators $x-f(y)$ and $x-g(y)$ do not depend on $x$. In the case where now $f(y)-g(y)$ is itself a valid denominator, this identity would therefore actually be a valid replacement rule and corresponds to a polynomial in the ideal $I$ in Eq. (9)). To ensure that this polynomial leads to the reduction (3), one can choose a monomial ordering, in which the inverse denominator variables $q_{1}$ and $q_{2}$ corresponding to $d_{1}=x-f(y)$ and $d_{2}=x-g(y)$, respectively, are "greater" than the inverse denominator variable $q_{3}$ corresponding to $d_{3}=f(y)-g(y)$. This motivates the use of a block ordering, in which we group all denominators depending on $x$ and $y$ and all denominators depending on only $x$ or only $y$.

Algorithm 3. Monomial block ordering. A suitable monomial ordering for the ring $K\left[q_{1}, \ldots, q_{m}, x_{1}, \ldots, x_{n}\right]$ in Algorithm 2 can be constructed as follows.

1. Group the denominators $d_{1}, \ldots, d_{m}$ by their dependence on all variables $x_{1}, \ldots, x_{n}$, such that denominators which depend on the same set of variables form a group; each group will correspond to a block in the monomial ordering. 
2. Sort the groups according to the number of variables they depend on; a group with denominators depending on fewer variables is considered "smaller" than a group with denominators depending on more variables.

3. In each group, sort the denominators according to their total degree.

4. Replace the denominators $d_{i}$ by the corresponding inverse denominator variables $q_{i}$ and add a last group containing the variables $x_{1}, \ldots, x_{n}$.

5. Let the sequence of groups of variables define the blocks of a monomial ordering. Within each block, use the "standard" degree reverse lexicographic ordering (degrevlex).

Let us consider at an example with the denominators

$$
d_{1}=x^{2}+y, d_{2}=x-y, d_{3}=x+1, d_{4}=x^{2}-3, d_{5}=y+1, d_{6}=y .
$$

The ideal $I$ is generated by the polynomials

$$
\left\{q_{1}\left(x^{2}+y\right)-1, q_{2}(x-y)-1, q_{3}(x+1)-1, q_{4}\left(x^{2}-3\right)-1, q_{5}(y+1)-1, q_{6} y-1\right\} .
$$

In steps $1-3$, we identify the three groups of denominators

$$
\left\{\left\{d_{1}=x^{2}+y, d_{2}=x-y\right\},\left\{d_{4}=x^{2}-3, d_{3}=x+1\right\},\left\{d_{5}=y+1, d_{6}=y\right\}\right\},
$$

which gives in step 4

$$
\left\{\left\{q_{1}, q_{2}\right\},\left\{q_{4}, q_{3}\right\},\left\{q_{5}, q_{6}\right\},\{x, y\}\right\} .
$$

This defines a monomial ordering with 4 blocks, see step 5 , which would be used to calculate the Gröbner basis and the subsequent polynomial reductions.

Our proposal for the monomial ordering aims to reduce the computational effort of the decomposition and to prefer low degrees of the denominator and numerator polynomials. In our experiments, it allowed for a significantly faster calculation of the Gröbner basis than a global degree ordering or a lexicographical ordering. We would like to point out that our choice of monomial order will in general only guarantee Leinartas' requirement (i) but not (ii). A lexicographical ordering could achieve a Leunartas decomposition fulfilling both requirements, but potentially at the price of significantly increased polynomial degrees. We give details and an example in Appendix B.

\subsection{Example for the algorithm}

We consider again the rational expression in Eq. (6),

$$
r=\frac{2 y-x}{y(x+y)(y-x)}
$$

and apply our partial fraction algorithm to it. We identify 3 irreducible denominators, and therefore consider the ideal

$$
I=\left\langle q_{1}(x-y)-1, q_{2} y-1, q_{3}(x+y)-1\right\rangle .
$$


Our monomial ordering is defined by

$$
\left\{\left\{q_{3}, q_{1}\right\},\left\{q_{2}\right\},\{x, y\}\right\} .
$$

and the resulting Gröbner basis is obtained as

$$
g=\left\{-1+q_{2} y,-1+q_{1} x-q_{1} y,-1+q_{3} x+q_{3} y,-q_{1} q_{2}+2 q_{1} q_{3}+q_{2} q_{3}\right\} .
$$

Reducing $r=(2 x-y) q_{1} q_{2} q_{3}$ with respect to $g$ yields the reduced form

$$
r=-\frac{1}{2} q_{1} q_{2}+\frac{3}{2} q_{2} q_{3}=\frac{3}{2 y(x+y)}+\frac{1}{2 y(y-x)},
$$

which is our partial fractioned result. Lets now assume we start with the equivalent expression from Eq. (8), i.e.

$$
r=\frac{1}{y(x+y)}+\frac{1}{(x-x)(x+y)}=q_{1} q_{2}+q_{2} q_{3} .
$$

Reducing this expression with respect to $g$ indeed yields exactly the same result as before, given by Eq. (18). Therefore, in this example, our algorithm recognizes that both input forms of $r$ are equivalent, and gives a unique result. If we however start in another form, in which spurious denominators occur, one has to be careful.

Let us consider a different representation of the same rational expression $r$ in (14), this time containing an additional spurious denominator:

$$
r=\frac{1}{y(x+y)}+\frac{1}{2 x} \frac{1}{(y-x)}-\frac{1}{2 x} \frac{1}{(x+y)} .
$$

In this case we would identify four denominators and consider the ideal

$$
I=\left\langle q_{1}(x-y)-1, q_{2} y-1, q_{3}(x+y)-1, q_{4} x-1\right\rangle .
$$

Our method produces in this case the block ordering

$$
\left\{\left\{q_{3}, q_{1}\right\},\left\{q_{2}\right\},\left\{q_{4}\right\},\{x, y\}\right\} .
$$

Calculating the corresponding Gröbner basis and reducing with respect to that basis yields

$$
r=-\frac{3}{2} q_{3} q_{4}-\frac{1}{2} q_{1} q_{4}+q_{2} q_{4} .
$$

We see that the spurious inverse denominator $q_{4}$ does not drop out, since we use a monomial ordering in which $q_{4} \prec q_{1}, q_{2}, q_{3}$. However, if we would have chosen a different monomial ordering, in which $q_{4}$ is greater than all other $q_{i}$, the spurious denominator would drop out after reduction. In this way, one can locally eliminate denominators which are known to be spurious or add additional denominators to the problem without altering previous reductions. These features are also supported by our package, cf. Section 4. 


\subsection{Efficient reduction of factorized inputs}

If the input expression is in a form with a single common denominator, i.e.

$$
r=q_{1}^{\alpha_{1} \ldots} q_{m}^{\alpha_{m}} \times \mathcal{N}
$$

where $\mathcal{N}$ is a possibly lengthy numerator, it may be optimal from a performance perspective to just fully expand the polynomial prior to reduction. In this case, we propose to the following guided scheme.

Algorithm 4. Iterated reductions. The polynomial reduction of $r$ in Eq. (24) with respect to $I$ can be performed as follows.

1. Set $p=\mathcal{N}$ and $Q=\left\{q_{1}^{\alpha_{1}}, \ldots, q_{m}^{\alpha_{m}}\right\}$.

2. Identify the "smallest" inverse denominator factor $q_{i}$ in $Q$ according to the monomial ordering defined in Section 3.2. Set $p \leftarrow p \times q_{i}^{\alpha_{i}}$ and remove $q_{i}^{\alpha_{i}}$ from $Q$.

3. Replace $p$ by its reduced form w.r.t. the Gröbner basis of the ideal $I$.

4. If $Q$ is non-empty, goto step 2. Otherwise, stop and return $p$.

The reason why this reduction scheme can be much faster than a "naive" direct reduction is, that the decomposed form of the common denominator $q_{1}^{\alpha_{1}}, \ldots, q_{m}^{\alpha_{m}}$ alone can result in huge expression, which only shortens once the numerator is taken into account. The iterative reduction of one denominator at a time avoids this intermediate expression swell, because it avoids the clustering of many denominators at one reduction step.

In the Mathematica functions of our package, the iterated scheme above can be selected as an option. Our Form implementation of the polynomial reductions uses this scheme by default. We observed an additional speed-up in our implementation in Mathematica by partitioning the whole expression into smaller pieces and reducing these individually in step 2. An example with a comparison of timings for different sizes of these partitions can be found in Section 5.3

\subsection{A comment on rational reconstructions}

Symbolic manipulations of large rational expressions can be computationally expensive. Finite field sampling and rational reconstruction techniques allow to prevent intermediate expression swell and have become quite standard in high energy physics calculations today [13, 14, 15, 16, 17, 18]. The basic idea is to set the indeterminates to numerical values in prime fields, perform all complicated manipulations with machine-sized integers instead of rational functions, and to reconstruct the final rational function of interest from many such samples. In this Section we would like to discuss the usage of information about the denominator structure for an improved reconstruction of rational functions. A major goal is to reduce the number of samples needed to reconstruct the rational functions, thus speeding up the computation. Furthermore, in applications involving linear relations between Feynman integrals, the partial fractioned forms 
are often particularly simple and it would be a significant advantage to directly reconstruct the partial fractioned form.

We first start with the following observation concerning the prediction of denominator factors in rational functions, which are to be reconstructed from numerical samples. Linear relations (integration-by-parts identities) between Feynman integrals typically involve only a small set of denominator factors. Moreover, choosing appropriate basis or master integrals can help to avoid spurious denominators in the calculation. In practice, the analysis of the denominator factors may be performed by considering only a small set of linear relations for a specific sector (set of distinct propagators) and setting subsector integrals (with fewer distinct propagators) to zero [19, 20]. This results in a list of possible denominator factors to be expected for a specific rational function. This knowledge helps to predict the denominator structure of the multivariate rational function e.g. by univariate reconstructions.

Here, we propose a new method, which allows to straight-forwardly guess denominator factors and their powers for a rational function, based on a list of candidate denominator factors and a small number of numerical probes (samples) of the rational function to reconstruct. Once the denominator is known or at least partial information about it, the full rational function can be reconstructed from (substantially) fewer samples. Let us assume we analyzed our rational functions and expect a list of irreducible denominator factors $d_{1}, \ldots, d_{m}$ in the result. We assume that the coefficient to be reconstructed is of the form

$$
r=\frac{\mathcal{N}}{d_{1}^{\alpha_{1}} \cdots d_{m}^{\alpha_{m}}},
$$

where $\mathcal{N}, d_{1}, \ldots, d_{m}$ are polynomials in $\mathbb{Q}\left[x_{1}, \ldots, x_{n}\right]$, and $\alpha_{1}, \ldots \in \mathbb{N}_{0}$.

Recipe 1. Denominator guess. For a rational function, which is expected to be of the form (25) with known denominator factors $d_{1}\left(x_{1}, \ldots\right), \ldots$, the following construction provides a guess for the full denominator.

1. Find integer samples for all $x_{1}, \ldots, x_{n}$ such that all $d_{1}, \ldots, d_{m}$ have a distinct, largest prime factor, which we denote by $p_{i}$, and each of these largest prime factors has multiplicity one. This requirement can be weakened but the stated form simplifies the following analysis.

2. Evaluate the rational function for the numerical values of the $x_{1}, \ldots, x_{n}$. In practice, this may mean e.g. linear system solving for several prime fields, Chinese remaindering and rational reconstruction. The result of this step is a number $r_{\text {num }} \in \mathbb{Q}$, which can be written in the form $r_{\text {num }}=$ $n_{\text {num }} / d_{\text {num }}$ with $n_{\text {num }}, d_{\text {num }} \in \mathbb{Z}$.

3. Perform a prime factor decomposition of the integer number $d_{\text {num. }}$. Due to Eq. (25), each factor $d_{i}^{\alpha_{i}}$ must contribute $p_{i}^{\alpha_{i}}$ to the prime factors in $d_{\text {num }}$. On the other hand, if we find a factor $p_{i}^{\alpha_{i}}$, we can take it as an indication that it was generated by $d_{i}^{\alpha_{i}}$ in the denominator. In this way, we can make a guess for the denominator just by counting the multiplicities of the $p_{i}$ in the factorization of $d_{\text {num }}$. 
In step 3 our guess may be off due the presence of a new denominator factor which was not in the candidate list or a factor $p_{i}$ appearing in the coefficients of the numerator polynomial $\mathcal{N}$. One could imagine to repeat the above construction for several samples of the indeterminates and combine this information to validate or correct the guess. In our experiments we have just picked somewhat larger integers and were in fact not even faced with these problems for the cases that we checked.

The above method lets us therefore identify (parts of) the denominator of the expression we want to reconstruct. A robust way to use this guess for the full reconstruction of a rational function is to multiply all samples with this denominator and reconstruct the resulting expression as if it was a rational function. If the guess was accurate, only the numerator needs to be reconstructed, thereby reducing the number of required samples. We emphasize that this method will allow for a successful reconstruction of the result even if the guess was incomplete or inaccurate. After reconstruction one can perform the partial fractioning as discussed in Section 3 .

We successfully applied this method to integration-by-parts reductions of Feynman integrals for problems with up to three loops. Considering the reconstruction of just a single variable, we typically observe a reduction of the number of samples by a factor of about 2 due to the guessed denominators. For a reduction problem with five variables, the iterated reconstruction of all variables resulted in an overall reduction of the number of samples by about a factor of 10 .

As a next step, one can ask if it is possible to directly reconstruct the result in partial fractioned form. Indeed, assuming one knows all irreducible denominator factors of an expression beforehand, our method in Section 3 actually allows to systematically construct a basis of all monomials that can be expected in the final partial fractioned result, using a bound on the monomial degree in the construction. This, in principle, allows to match against an ansatz and reconstruct the partial fractioned form directly. We observed in our experiments that depending on the exact strategy the ansatz in partial fractioned form may require more samples to fix the free coefficients than the reconstruction in the common denominator representation along the lines described above. Furthermore, an incomplete list of denominator factors would prevent a successful fit of a partial fractioned ansatz as well as an insufficient degree of the ansatz. In contrast, the method for the reconstruction in common-denominator form presented above is robust with respect to incomplete denominator information.

\section{The package}

\subsection{Installation and basic usage}

We implement the described algorithm in a Mathematica package, that can be downloaded as follows:

git clone https://gitlab.msu.edu/vmante/multivariateapart.git 
The package consists of just a single file, MultivariteApart.wl, which could also be downloaded individually. We recommend to place it in a standard Mathematica packages directory, e.g. /. Mathematica/Applications, such that it can be loaded with

$$
\operatorname{In}[1]:=\text { Needs ["MultivariateApart" "] }
$$

from any directory. Alternatively, it can always be loaded by specifying the full path to the file. The main function, which implements the algorithm described in Section 3 can be called by MultivariateApart. Using this command on the example of Section 3.3 yields:

$$
\begin{aligned}
& \ln [2]:=\text { MultivariateApart }[(2 y-x) /(y(x+y)(y-x))] \\
& \text { Out }[2]=-\frac{1}{2(x-y) y}+\frac{3}{2 y(x+y)}
\end{aligned}
$$

The function provides a unique representation and may be all that is needed in many cases.

It can be convenient to keep the inverse factors abbreviated for further processing, which can be achieved by using MultivariateAbbreviatedApart instead:

$$
\begin{aligned}
\ln [3]:= & \text { MultivariateAbbreviatedApart }[(2 y-x) /(y(x+y)(y-x))] / / \text { Normal } \\
\text { Out }[3]= & \{-((q 1 \mathrm{q} 2) / 2)+(3 \mathrm{q} 2 \mathrm{q} 3) / 2,\{\mathrm{q} 1 \rightarrow 1 /(\mathrm{x}-\mathrm{y}), \mathrm{q} 2 \rightarrow 1 / y, \\
& \mathrm{q} 3 \rightarrow 1 /(\mathrm{x}+\mathrm{y})\}\}
\end{aligned}
$$

Here, Normal was used to convert the dispatch table in the second element of the returned list into normal form. One can pass a list of previously identified denominator factors as a second argument to MultivariateAbbreviatedApart:

$$
\ln [4]:=\text { MultivariateAbbreviatedApart }[(2 y-x) /(y(x+y)(y-x)),\{x-y, y, x+y\}]
$$

This allows one to work with a global list of abbreviations across several expressions.

The package provides further functions and options, which may be useful for more granular control, performance tuning and interfacing with other computer algebra systems. We will describe some of this functionality in the following. Please see the output of ?MultivariateApart'* for a full list of available functions and options.

\subsection{Advanced usage pattern}

The "all-in-one" functions described so far internally call a sequence of other functions, which can also be used directly for more advanced use cases. With default options, the first step is a call to Together to eliminate spurious denominators. This behavior as well as other details can be configured through the options of the function. Of course, for our example this step is irrelevant. Next, AbbreviateDenominators is called, which finds all denominators in an expression and replaces them by a predefined symbol (the default is $q_{i}$ ).

$$
\begin{aligned}
& \operatorname{In}[5]:=\{\operatorname{expr}, \text { dens, qis }\}=\text { AbbreviateDenominators }[(2 y-x) /(y(x+y)(y-x)] \\
& \text { Out }[5]=\{-q 1 \text { q2 q3 }(-x+2 y),\{x-y, y, x+y\},\{q 1, q 2, q 3\}\}
\end{aligned}
$$


The output is a list, whose first entry is the expression in terms of the denominator symbols, the second entry a list of all irreducible denominator factors and the last entry a list of their abbreviations. Optionally, AbbreviateDenominators accepts a list of denominator factors as a second argument to support working with a global list of abbreviations. Next, Apart0rder is used to define the monomial order:

$$
\begin{aligned}
\operatorname{In}[6]:=\operatorname{ord}=\operatorname{ApartOrder}[\text { dens, } & \mathrm{qis}] \\
\operatorname{Out}[6] & =\{\{\mathrm{q} 3, \mathrm{q} 1\},\{\mathrm{q} 2\},\{\mathrm{x}, \mathrm{y}\}\}
\end{aligned}
$$

The returned list represents the monomial order, that will be used for the Gröbner basis computation, cf. Section [3.2. The Gröbner basis is obtained with

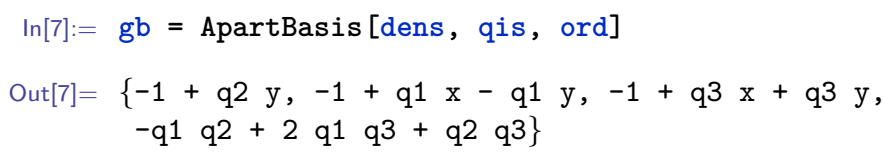

The actual reduction or partial fractioning is performed with

$$
\begin{aligned}
& \operatorname{In}[8]:=\text { ApartReduce [expr, gb, ord }] \\
& \text { Out[8] }=-\frac{\mathrm{q} 1 \mathrm{q} 2}{2}+\frac{3 \mathrm{q} 2 \mathrm{q} 3}{2}
\end{aligned}
$$

This example also shows the alternative way of using our package. Instead of using MultivariateApart on a single expression, we can think about the situation where we know all denominators of a set of expressions beforehand, and then want to bring a give expression into a unique form. Lets assume we have an additional denominator $x$ in the problem. We can then first calculate the Gröbner base including this additional denominator and then reduce with respect to that base. As we have seen already in Section 3.3 there is no guarantee that an expression is free of spurious singularities in this case:

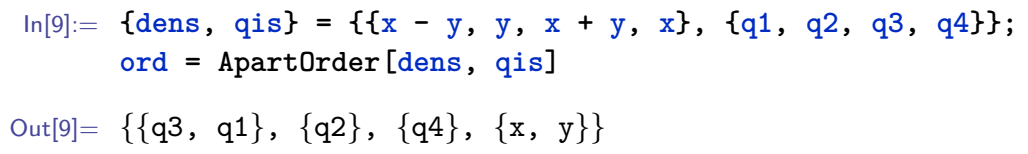

Note that the algorithm considers $q_{4}=1 / x$ to be "simple" and will try to eliminate the other $q_{i}$ in favor of $q_{4}$.

$$
\begin{aligned}
\ln [10]:= & g b=\text { ApartBasis [dens, qis, ord] } ; \\
& \text { ApartReduce [expr, gb, ord] } \\
\text { Out }[10]= & -\frac{q 1 q^{4}}{2}+q 2 q 4-\frac{3 q 3 q 4}{2}
\end{aligned}
$$

Here, the pole $q_{4}=1 / x$ is actually spurious.

If we knew already beforehand that $q_{4}$ is spurious, we could have used a monomial order such that $q_{4}$ drops out (one could think of a situation, in which one knows that some denominators should not occur in the final answer). In this example, this can be done by changing the list ord in the following way: we just reorder the blocks in ord by moving the block with $q_{4}$ to the left most position (i.e. that block is greater than all other): 


$$
\begin{aligned}
\ln [11]:= & \operatorname{ord}=\{\{q 4\},\{q 3, q 1\},\{q 2\},\{x, y\}\} \\
& g b=\text { ApartBasis [dens, qis, ord]; } \\
& \text { ApartReduce [expr, gb, ord] } \\
\text { Out[11]= } & -\frac{q 1 q^{2}}{2}+\frac{3 q 2 q^{3}}{2}
\end{aligned}
$$

As expected, the result is free of the spurious pole $q_{4}$ and we find the same answer, as using MultivariateApart directly on the whole expression. Let us demonstrate the important fact that, once a monomial order is chosen, the final answer is essentially insensitive to the exact input form, and one can equally well operate on individual terms of a sum:

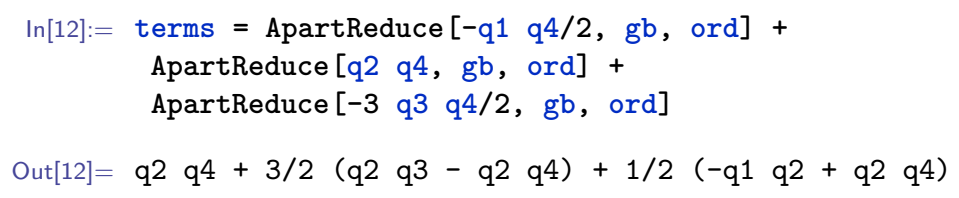

Note how in this case the partial fractioned form of the invidual terms actually exhibit the pole $q_{4}$, but they cancel in the sum after a call to Expand,

$$
\begin{aligned}
& \ln [13]:=\text { Expand[terms] } \\
& \text { Out[13]}=-\frac{\mathrm{q} 1 \mathrm{q} 2}{2}+\frac{3 \mathrm{q} 2 \mathrm{q} 3}{2}
\end{aligned}
$$

which is the same result as before.

\subsection{Interface with Singular}

Although our package can be used entirely in Mathematica, for difficult examples we recommend to perform the computation of the Gröbner basis in Singular. An input file for the calculation of the Gröbner basis can be generated with the function WriteSingularBasisInput, which can be executed in Singular with the read() function. As an example how to use Singular with our package we return to the previous example:

$$
\begin{aligned}
& \ln [14]:=\{\text { expr, dens, } q \text { is }\}=\text { AbbreviateDenominators }[(2 y-x) /(y(x+y)(y-x)] \\
& \text { Out }[14]=\{-q 1 \text { q2 } q 3(-x+2 y),\{x-y, y, x+y\},\{q 1, q 2, q 3\}\} \\
& \ln [15]:=\text { ord }=\text { ApartOrder }[\text { dens, qis }] \\
& \text { Out }[15]=\{\{q 3, q 1\},\{q 2\},\{x, y\}\}
\end{aligned}
$$

We can now prepare the Gröbner basis calculation with the command

$$
\ln [16]:=\text { WriteSingularBasisInput [dens, qis, ord, "dir"] }
$$

Here, "dir" denotes the name of the directory to which input and output files are written. The specified directory will be created if it does not exist. All commands for Singular are stored in the input file apartbasisin.sing. In Singular we can use execute(read(apartbasisin.sing)) to execute these commands. After execution, the file apartbasisout.m is created, which contains the Gröbner basis. We can load it in Mathematica: 


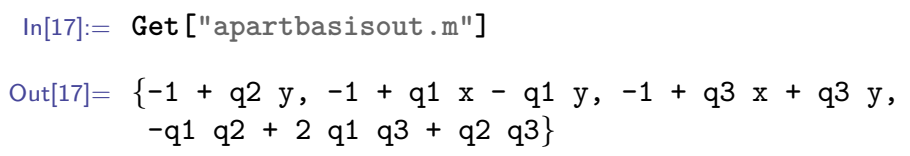

This is the same result as before when we used ApartBasis in the previous Section.

We note that Singular provides support for polynomial reductions and can therefore also be used instead of our function ApartReduce. We observed good timings in this approach for some of our more complicated examples.

\subsection{Interface with Form}

We emphasize that the polynomial reduction step of our algorithm allows a straight forward implementation in symbolic manipulation programs such as Form, in which one uses identify statements to "locally" replace parts of expressions, that is, one considers one term of a potentially large sum at a time. Having calculated the Gröbner basis, these identify statements can be derived by considering the leading term of each generator and generating rules that replace these leading terms. We have successfully used our methods in this way and added the function WriteFormProcedure in our package. It can be invoked by

$\ln [18]:=$ WriteFormProcedure [gb, ord, "dir"]

Here, $\mathrm{gb}$ is the Gröbner base from which the replacement rules are derived, ord is the monomial order that was used and "dir" is the directory where the Form procedure shall be written to. The command generates a Form input file called "apartreduce.h", containing a procedure that implements the reduction scheme described in Section 3.4. and two auxiliary files, which have to be in the same directory. For usage in Form, the file has to be included in the beginning of a Form script with "\#include apartreduce.h" and the reduction algorithm can be invoked by "\#call apartreduce(expr)", where expr is a local Form expression. Note that "apartreduce.h" contains the definition of the denominator symbols in a specific order, which is needed for an efficient reduction. The user should therefore not define these symbols at another point in his or her Form scripts.

It is also possible to use Form as a back end for the computation of polynomial reductions in MultivariateApart, MultivariateAbbreviatedApart, and ApartReduce by specifying the option UseFormProgram $\rightarrow$ True. For example, the command

$\ln [19]:=$ ApartReduce [expr, gb, ord, UseFormProgram $\rightarrow$ True]

calls the external program Form to perform the actual computation. Here, the Form executable is assumed to be in the user's path, and the directory \$ApartTemporaryDirectory will be used for all temporary files. 


\section{Applications}

The methods described in this paper have been successfully applied to several calculations of Feynman amplitudes. In this Section, we provide some details for these applications.

\subsection{One-loop amplitudes for $\gamma^{*} \gamma^{*} \rightarrow l^{+} l^{-}$with finite lepton mass}

One of us calculated all next-to-leading-order helicity amplitudes for the process

$$
\gamma^{*}\left(p_{1}\right)+\gamma^{*}\left(p_{2}\right) \rightarrow l^{+}\left(p_{3}\right)+l^{-}\left(p_{4}\right),
$$

where the quantities in parenthesis denote the four-momenta of the particles. The calculation [21, 22] included the full dependence on the lepton mass using the ideas of this paper. Defining the kinematic quantities

$$
p_{1}^{2}=t_{1}, \quad p_{2}^{2}=t_{2}, \quad p_{3}^{2}=p_{4}^{2}=m^{2}, \quad\left(p_{1}+p_{2}\right)^{2}=s, \quad\left(p_{1}-p_{3}\right)^{2}=t,
$$

and setting $m^{2} \equiv 1$, one encounters a total of 15 denominators, for which our package finds the optimized ordered form

$$
\begin{aligned}
& \left\{\left\{s-2 s t+s^{2} t+s t^{2}-s t_{1}-s t t_{1}+t_{1}^{2}-s t_{2}-s t t_{2}-2 t_{1} t_{2}+s t_{1} t_{2}+t_{2}^{2},\right.\right. \\
& 1-2 s+s^{2}-2 t+2 s t+t^{2}-4 t_{1}+2 t_{2}-2 s t_{2}-2 t t_{2}+t_{2}^{2}, \\
& 1-2 s+s^{2}-2 t+2 s t+t^{2}+2 t_{1}-2 s t_{1}-2 t t_{1}+t_{1}^{2}-4 t_{2}, \\
& \left.2-s-t+t_{1}+t_{2}, 1-s-t+t_{1}+t_{2}\right\},\left\{s^{2}-2 s t_{1}+t_{1}^{2}-2 s t_{2}-2 t_{1} t_{2}+t_{2}^{2},\right. \\
& \left.s-t_{1}-t_{2}\right\},\left\{1-2 t+t^{2}-2 t_{2}-2 t t_{2}+t_{2}^{2}\right\},\left\{1-2 t+t^{2}-2 t_{1}-2 t t_{1}+t_{1}^{2}\right\} \\
& \left.\{t,-1+t\},\{s, 4-s\},\left\{t_{2}\right\},\left\{t_{1}\right\}\right\} .
\end{aligned}
$$

Using the block ordering described in Section 3.2, we can calculate the Gröbner basis for the ideal formed from these denominators using slimgb in Singular in a few minutes.

It is worth noting, that three of these denominators are spurious and result as an artefact of the basis of Lorentz invariant building blocks, that are used to build the amplitudes [23]. Namely, the three denominators that should drop out in the end are

$$
t_{1}, t_{2}, s-t_{1}-t_{2} .
$$

Changing the monomial order slightly, such that these denominators form an extra block which is "greater" than all other denominators, we are still able to calculate the Gröbner basis of this ideal. We furthermore find that in the final answer these denominators indeed cancel out.

Comparing the size of the results in factorized form and partial fractioned form, we find a reduction by factor 7 on average. However, some individual amplitudes are reduced by a factor of 20 to 30 . 


\subsection{Two-loop amplitudes for $g g \rightarrow Z Z$ with a closed top-quark loop}

The algorithm presented in this work has also been applied to the calculation of the two-loop QCD corrections to the process

$$
g\left(p_{1}\right)+g\left(p_{2}\right) \rightarrow Z\left(p_{3}\right)+Z\left(p_{4}\right)
$$

involving a closed top-quark loop in Ref. [24]. In that calculation,

$$
p_{1}^{2}=p_{2}^{2}=0, \quad p_{3}^{2}=p_{4}^{2}=m_{z}^{2}, \quad\left(p_{1}+p_{2}\right)^{2}=s, \quad\left(p_{1}-p_{3}\right)^{2}=t,
$$

was used for the kinematics and the masses of the top quark and the $Z$ boson, $m_{t}$ and $m_{z}$, have been fixed by $m_{t}=1$ and $\left(m_{z} / m_{t}\right)^{2}=5 / 18$. It is possible to choose a basis of master integrals, such that the dependence on the space-time dimension $d$ factorizes from denominators involving the kinematic invariants $s$ and $t$. The coefficients contain the $9 d$-dependent denominators

$$
\{5 d+52,3 d-10,3 d-8,2 d-7, d-5, d-4, d-3, d-2, d\}
$$

and the $48 d$-independent denominators

$$
\begin{aligned}
& \left\{\left\{2125764 t^{6}+8503056 s t^{5}-5904900 t^{5}+12754584 s^{2} t^{4}-35901792 s t^{4}\right.\right. \\
& +2001105 t^{4}+8503056 s^{3} t^{3}-72275976 s^{2} t^{3}+25850340 s t^{3}+3863700 t^{3} \\
& +2125764 s^{4} t^{2}-60466176 s^{3} t^{2}+75149694 s^{2} t^{2}-19260180 s t^{2}-797850 t^{2} \\
& -18187092 s^{4} t+80752788 s^{3} t-73614420 s^{2} t+17309700 s t-468000 t \\
& +29452329 s^{4}-50490540 s^{3}+25891650 s^{2}-3676500 s+105625,26244 t^{6} \\
& +52488 s t^{5}-14580 t^{5}+26244 s^{2} t^{4}-224532 s t^{4}-56295 t^{4}-209952 s^{2} t^{3} \\
& +116640 s t^{3}+32400 t^{3}+419904 s^{2} t^{2}-249480 s t^{2}+27900 t^{2}+64800 s t \\
& -18000 t+2500,524880 t^{4}+524880 s t^{3}-583200 t^{3}+1889568 s^{2} t^{2} \\
& +1953720 s t^{2}+243000 t^{2}+3149280 s^{2} t-1206900 s t-45000 t+145800 s^{2} \\
& +173250 s+3125,104976 t^{4}+314928 s t^{3}-536544 t^{3}+209952 s^{2} t^{2} \\
& -1405512 s t^{2}+398520 t^{2}-839808 s^{2} t+599400 s t-106200 t-64800 s+9625, \\
& 104976 t^{4}+104976 s t^{3}+303264 t^{3}-104976 s^{2} t^{2}+29160 s t^{2}-301320 t^{2} \\
& -104976 s^{3} t-244944 s^{2} t+68040 s t+88200 t+29160 s^{3}-32400 s^{2}-23400 s \\
& -8375,13122 s t^{3}+37908 t^{3}+26244 s^{2} t^{2}+64881 s t^{2}-56700 t^{2}+13122 s^{3} t \\
& +26973 s^{2} t-52650 s t+22725 t+2025 s^{2}+1800 s-2750,104976 s^{2} t^{2} \\
& -898128 s t^{2}+1454436 t^{2}+209952 s^{3} t-1073088 s^{2} t+64800 s t-808020 t \\
& +104976 s^{4}-174960 s^{3}-144180 s^{2}+51300 s+112225,104976 s^{2} t^{2} \\
& -898128 s t^{2}+1454436 t^{2}-839808 s^{2} t+3841992 s t-808020 t+1679616 s^{2} \\
& -997920 s+112225,324 s^{2} t^{2}-2592 s t^{2}+5184 t^{2}+648 s^{3} t-2772 s^{2} t \\
& +1440 s t-2880 t+324 s^{4}-180 s^{3}-695 s^{2}-200 s+400,324 s^{2} t^{2}-2592 s t^{2}
\end{aligned}
$$




$$
\begin{aligned}
& +5184 t^{2}-2772 s^{2} t+11808 s t-2880 t+4489 s^{2}-3080 s+400,81 s t^{2}-324 t^{2} \\
& +162 s^{2} t-414 s t+180 t+81 s^{3}-90 s^{2}+25 s-25,81 s t^{2}-324 t^{2}-324 s t \\
& +180 t-25,3015 t^{2}+3015 s t-1675 t+2916 s^{2}-6030 s+3350,324 t^{2} \\
& +648 s t-180 t+25,324 t^{2}+558 s t-180 t+324 s^{2}-155 s+25,324 t^{2} \\
& +324 s t-180 t-1296 s+25,324 t^{2}+324 s t-180 t+25,324 t^{2}+90 s t-180 t \\
& +90 s^{2}-25 s+25,324 t^{2}-180 t-324 s^{2}+180 s+25,162 t^{2}+162 s t-135 t \\
& +25, \quad 18 t^{2}+18 s t-5 t+5 s, 558 s t-90 t+558 s^{2}-580 s+25,558 s t-90 t \\
& +180 s+25,324 s t-90 t+324 s^{2}-270 s+25,324 s t-90 t+25,279 t \\
& +279 s-200,117 t+117 s-155,18 t+36 s-5,18 t+18 s-5,18 t+9 s \\
& -5,18 t-18 s-5,9 t+9 s+31,9 t+9 s-5,6 t+6 s-5\},\left\{324 s^{2}-245 s\right. \\
& +90,324 s-335,18 s-5,9 s+134,9 s-5,9 s-10, s-4, s\},\{31 t+5 \text {, } \\
& 18 t+5, \quad 18 t-5,13 t+10, t-4, t\}\} .
\end{aligned}
$$

During the course of the calculation, different choices for the master integrals were used, and the original choices did in fact introduce additional denominators, which mixed the dependence on $d$ and the kinematic invariants. After changing to an improved set of master integrals, the methods presented in this paper allowed to systematically eliminate those denominators in a term-by-term approach and to very substantially reduce the size of the expressions. In order to reduce the computational complexity, the partial fraction decomposition was performed for expressions expanded around $d=4$, allowing to effectively replace $d$ by 4 in the denominator analysis. The actual polynomial reduction was performed directly in Singular in this case.

The final result for this amplitude was rather sizable. However, the partial fractioned form of the rational coefficients of the master integrals allowed to conveniently generate fast code for their numerical evaluation. Starting from a form with symbols for the inverse denominator factors, also powers of inverse denominator factors and of basic invariants were abbreviated. In this way, each coefficient becomes a sum of simple products of a few factors each, where the basic factors (the powers) can be precomputed numerically. This allows to straight-forwardly generate compiler friendly code, where each term is added to a summation variable numerically.

\subsection{Two-loop amplitudes for massless five-particle scattering}

For a comparison with the algorithm presented in Ref. [12], we applied our algorithm to a benchmark example considered in that work: the integrationby-parts matrix provided in Ref. [25] for the reduction of two-loop five-point functions in terms of a dlog basis. The kinematic variables are chosen as

$$
c_{2}=s_{23} / s_{12}, \quad c_{3}=s_{34} / s_{12}, \quad c_{4}=s_{45} / s_{12}, \quad c_{5}=s_{15} / s_{12},
$$

where $s_{12}=1$ and the $s_{i j}$ are Lorentz invariant functions of the external momenta. Furthermore, $\epsilon$ is the parameter of dimensional regularization. In the 


\begin{tabular}{|l|r|}
\hline Option & Runtime \\
\hline Iterate->False (default) & $9457 \mathrm{sec}$ \\
Iterate->True PartionSize-> $\infty$ & $9254 \mathrm{sec}$ \\
Iterate->True, PartionSize->5000 & $635 \mathrm{sec}$ \\
Iterate->True, PartionSize->2000 & $422 \mathrm{sec}$ \\
Iterate->True, PartionSize->1000 & $358 \mathrm{sec}$ \\
Iterate->True, PartionSize->100 & $489 \mathrm{sec}$ \\
UseFormProgram->True & $60 \mathrm{sec}$ \\
\hline
\end{tabular}

Table 1: Timing of MultivariateApart applied to entry $(1,16)$ of the matrix provided in 25] for different options.

matrix of rational functions, we encounter a total of 24 denominators 1 ,

$$
\begin{aligned}
\{ & -1+c_{3}, c_{3}, c_{2}, 1+c_{2}, c_{2}+c_{3}, c_{2}-c_{4}, 1+c_{2}-c_{4},-1+c_{4}, c_{4},-1+c_{3}+c_{4}, \\
& c_{3}+c_{4}, c_{2}-c_{5},-1+c_{3}-c_{5}, c_{2}+c_{3}-c_{5}, c_{2}-c_{4}-c_{5}, 1+c_{2}-c_{4}-c_{5} \\
& -1+c_{3}+c_{4}-c_{5}, c_{5}, c_{2}^{2}-2 c_{2}^{2} c_{3}+c_{2}^{2} c_{3}^{2}+2 c_{2} c_{3} c_{4}-2 c_{2} c_{3}^{2} c_{4}+c_{3}^{2} c_{4}^{2}-2 c_{2} c_{5} \\
& +2 c_{2} c_{3} c_{5}+2 c_{2} c_{4} c_{5}+2 c_{3} c_{4} c_{5}+2 c_{2} c_{3} c_{4} c_{5}-2 c_{3} c_{4}^{2} c_{5}+c_{5}^{2}-2 c_{4} c_{5}^{2}+c_{4}^{2} c_{5}^{2} \\
& -1+\epsilon,-1+2 \epsilon,-1+3 \epsilon,-1+4 \epsilon, 1+4 \epsilon\} .
\end{aligned}
$$

First, we apply MultivariateApart to the rational function appearing as the $16^{\text {th }}$ entry of the first row of the matrix and study the impact of different options on the runtime. As can be seen in Table 1, different options can alter the performance significantly despite producing the same output form. In the Mathematica-only approach, enabling the iterated reduction scheme described in Section 3.4 can help significantly to reduce the runtime, provided one selects a suitable finite partition size in addition. We don't see a general argument why the partitioning should have the observed effect in all cases; we are tempted to speculate that finite internal buffer sizes in Mathematica could be the reason here. Enabling the flag to use Form instead of Mathematica for the polynomial reductions results in the smallest runtime by quite a margin in this example. We note that the Form implementation also uses iterated reduction approach of Section 3.4

Next, we consider the partial fraction decomposition of all entries of the benchmark matrix in Ref. 25]. We ran our algorithm in two different setups, once by performing the partial fractioning of each term independently with a Gröbner basis containing only denominators that appear in that term, and once with a global Gröbner basis containing all 24 denominators. Note that these two approaches result in well defined but different output forms. In both cases, we enable the option of our package to reduce the polynomials with Form, which was essential to achieve the best timing. In Table 2 we list the resulting timings of

\footnotetext{
${ }^{1}$ Note that the reduced matrix given in Ref. [12] has 32 denominators because several appear twice modulo a sign change.
} 


\begin{tabular}{|l|c|c|c|c|}
\hline Algorithm & Runtime & File size & Max. mon. deg. & Max. term length \\
\hline Ref. [12] & & $25.1 \mathrm{MB}$ & 20 & 3564 \\
Global GB & $863 \mathrm{~min}$ & $22.6 \mathrm{MB}$ & 12 & 3109 \\
Local GB & $356 \mathrm{~min}$ & $21.3 \mathrm{MB}$ & 12 & 3048 \\
\hline
\end{tabular}

Table 2: Comparison between our algorithm in the local and global reduction approach and the algorithm in Ref. [12]

these runs and compare features of the resulting output forms to those obtained with the algorithm of 12 .

The local Gröbner basis approach is most comparable to the algorithm used in Ref. [12]. With this setup, our algorithm finished the partial fraction decomposition of all elements of the benchmark matrix in roughly 5.5 hours on a single core of a desktop computer. For the approach in which one calculates one global Gröbner basis for all 24 denominators at once, our algorithm finds an optimized monomial ordering with which the Gröbner basis can be calculated within one minute in Mathematica. In this approach the reduction of all matrix elements took roughly 14.4 hours on a Desktop computer, which is longer than in the local Gröbner basis approach. We did not attempt to make a serious comparison of performance to the implementation of [12], but the timing for this example seems to be of a similar order of magnitude compared to the runs of our package (Ref. 12] reported 27.9 hours of runtime).

The resulting output forms are of similar overall size in all cases, see Table 2 Considering the rational functions as polynomials in $\mathbb{Q}\left[q_{1}, \ldots, q_{24}, c_{2}, \ldots, c_{5}, \epsilon\right]$, we determine the total degrees of the output forms in the different approaches. In all cases, we consider fully expanded output forms and write them to disk with Mathematica. As is shown in Table 2, our method leads to lower degrees than the decomposition of Ref. [12], as expected due to our choice of monomial ordering, cf. Section 3.2 For the comparison, it is noteworthy that our package does not treat non-linear denominators in a special way, they are fully taken into account in the decomposition. Using a global Gröbner basis for the reduction yields a slightly larger result but has the big advantage that all terms are not only in a unique representation locally, but also globally. Therefore sums of different coefficients which would appear in the calculation of the amplitude can be computed without any problems term by term, e.g. using Form.

\section{Conclusion and outlook}

In this paper we introduced a method for the partial fraction decomposition of multivariate rational functions through polynomial reductions. The method does not introduce spurious singularities and allows for a straightforward implementation in Form using local replacement rules. We implemented our algorithms in a Mathematica package that is publicly available at https://gitlab.msu.edu/vmante/multivariateapart

Our approach involves the construction of a special monomial ordering, which is optimized for computational performance and leads to results with 
comparably low degrees. We showed for different "real life" examples how the algorithm helped to calculate Feynman amplitudes, in the presence of many kinematic variables, many denominators, or high polynomial degrees of the denominators. In several of our examples, the calculation of the Gröbner basis is highly non-trivial and became possible through our method to define the monomial ordering. The partial fractioned sum of terms lends itself to a straightforward and compiler friendly implementation in numerical codes.

We discussed options for the reconstruction of rational functions from prime field samples. Here, we proposed a new idea to reconstruct the denominator of a rational function based on knowledge about possible denominator factors and a small number of numerical samples. This method has been successfully tested in the context of linear relations between Feynman integrals and reduced the number of samples required for the reconstruction of the full rational functions significantly. Our partial fraction decomposition provides a systematic approach to the direct reconstruction rational function in this representation. Future research might provide an optimized strategy to further reduce the number of samples required to reconstruct complicated rational functions in this approach.

Another future application of our methods could be the direct parametric integration of Feynman integrals [26, 27]. In this context it is important to recognize potential cancellations between different terms and to avoid the introduction of spurious (non-linear) denominators, since they can prevent a successful integration. The algorithms discussed in this paper provide a systematic approach to this problem.

\section{Acknowledgements}

We would like to thank Marco Besier, Bakul Agarwal and Federico Buccioni for helpful discussions and valuable feedback on applications of our package. MH was supported in part by the German Research Foundation (DFG), through the Collaborative Research Center, Project ID 204404729, SFB 1044, and the Cluster of Excellence PRISMA+, Project ID 39083149, EXC 2118/1. AvM was supported in part by the National Science Foundation under Grants No. 1719863 and 2013859.

\section{A. Polynomial reductions}

In this Appendix, we review some basic notions that can be found in any introductory textbook on the topic, see e.g. [10, 11].

Let us consider polynomials in the variables $x_{1}, \ldots, x_{n}$ with coefficients in the field $K$ (e.g. the rational numbers). These polynomials form a polynomial ring $R=\mathbf{K}\left[x_{1}, \ldots, x_{n}\right]$.

A monomial ordering is a total order $\prec$ on the set of monomials

$$
M \equiv\left\{x^{\alpha} \equiv x_{1}^{\alpha_{1}} \cdots x_{n}^{\alpha_{n}} \mid \alpha_{i} \in \mathbf{Z}_{\geq 0}\right\}
$$

such that 
(i) for all monomials $x^{\alpha}, x^{\beta}$ and $x^{\gamma}: x^{\alpha} \leq x^{\beta} \Rightarrow x^{\alpha+\gamma} \leq x^{\beta+\gamma}$,

(ii) $1 \leq x^{\alpha} \quad \forall \alpha$.

In many applications, in which the specific monomial ordering does not matter, one chooses the so-called degree reverse lexicographic order (degrevlex), which first compares the total degree of two monomials, and then, if the degrees are the same, uses a lexicographic comparison and reverses the outcome of the latter.

There is a more general way of defining a monomial ordering, which will be relevant to us. A monomial ordering is called a block ordering, if one can group the variables into two sets $\left\{x_{1}, \ldots, x_{m}\right\}$ and $\left\{y_{1}, \ldots, y_{n}\right\}$, such that

$$
x^{\alpha} \prec y^{\beta} \quad \forall \alpha, \beta \text { with } y^{\beta} \neq 1
$$

i.e. all monomials formed from variables from the first block are "smaller" than monomials formed from variables from the second block.

We call the "greatest" monomial of a polynomial $p$ with respect to a monomial order the leading monomial of p. A term is a monomial multiplied with a coefficient, which is a non-zero rational number for our purposes. The leading term $\operatorname{LT}(p)$ of polynomial $p$ is the term corresponding to the leading monomial.

Having defined a monomial ordering puts us in a position to discuss polynomial reductions. Given two polynomials $p_{1}$ and $p_{2}$ together with a monomial ordering $\prec$, we call $p_{1}$ reducible modulo $p_{2}$ if for some term $t$ of $p_{1}$ there is a term $u$ such that $t=u \cdot \operatorname{LT}\left(p_{2}\right)$. Then we say, $p_{1}$ reduces to $p_{1}^{\prime}$ modulo $p_{2}$, where

$$
p_{1}^{\prime}=p_{1}-u \cdot p_{2}
$$

The effect of the polynomial reduction is that a term in $p_{1}$ is replaced by a linear combination of "smaller" terms.

It is useful to introduce another concept. A set of polynomials $g_{1}, \ldots g_{m} \in R$, the generators, define an ideal $I \subset R$ as the set of all their linear combinations, where the coefficients are polynomials themselves, $I=\left\{\sum_{i} f_{i} g_{i}\right.$ with $\left.f_{i} \in R\right\}$. We also use the notation $I=\left\langle g_{1}, \ldots, g_{m}\right\rangle$. The choice of generators is not unique, and it is a non-trivial task to decide whether a polynomial is a member of a given ideal. It is clear that, if a polynomial reduces to zero modulo the generators of an ideal that the polynomial is a member of that ideal. The inverse, however, is not true and the remainder of a polynomial reduction will in general depend on the individual reduction steps.

It is possible to find a set of generators for an ideal, called a Gröbner basis, which allows one to find a unique remainder for the reduction of any polynomial modulo the generators. In this case, a reduction to zero occurs if and only if the polynomial is in the ideal. A Gröbner basis can be calculated algorithmically by adjoining specific differences of generators. The Gröbner basis depends on the choice of the monomial ordering and is unique if reduced with respect to itself. 


\section{B. LeǏnartas' requirements and polynomial reductions}

In this Section we revisit the two central decomposition steps in Algorithm 1 leading to Leĭnartas' decomposition, see Section 2.2 and consider under which circumstances they are reproduced by the polynomial reductions in Algorithm 2 , see Section 3. As we will show, Algorithm 2 may or may not produce a Leĭnartas' decomposition, depending on the monomial ordering. If the monomial ordering sorts first for the $q_{i}$ and then for the $x_{i}$, requirement (i) will be filfilled, that is, the denominator zeros will be separated. If the $q_{i}$ are sorted lexicographically, also requirement (ii), the algebraic independence of different denominator factors, will be guaranteed [9]. For degree based orderings of the $q_{i}$, requirement (ii) is violated in general. This means in particular, that our monomial block ordering proposed in Section 3.2 guarantees only requirement (i) and not (ii), and will therefore not lead to a Leinartas decomposition. We emphasize, that an even more general choice of monomial ordering will ensure neither of the two requirements, but still allows for a unique output form.

First, we consider the requirement (i), i.e. that the denominators of each term shall have common zeros in $\bar{K}^{n}$. Let us assume we encounter a term which is not fully decomposed yet and has denominators $\left\{d_{1}\left(x_{1}, \ldots\right), \ldots, d_{m}\left(x_{1}, \ldots\right)\right\}$. By Hilbert's Nullstellensatz, a finite set of polynomials $\left\{d_{1}^{\alpha_{1}}, \ldots, d_{m}^{\alpha_{m}}\right\}$ has no common zeros in $\bar{K}^{n}$ if and only if there exist polynomials $h_{1}, \ldots, h_{m}$, such that

$$
1=\sum_{i} h_{i}\left(x_{1}, \ldots\right) d_{i}^{\alpha_{i}}\left(x_{1}, \ldots\right) .
$$

Dividing this equation by $d_{1}^{\alpha_{1}} \cdots d_{m}^{\alpha_{m}}$ gives the decomposition [6]

$$
\frac{1}{d_{1}^{\alpha_{1}} \cdots d_{m}^{\alpha_{m}}}=\sum_{i} \frac{h_{i}\left(x_{1}, \ldots\right)}{d_{1}^{\alpha_{1}} \cdots \hat{d}_{i}^{\alpha_{i}} \cdots d_{m}^{\alpha_{m}}} .
$$

where $\hat{d}_{i}^{\alpha_{i}}$ means leaving $d_{i}^{\alpha_{i}}$ out in the product.

This decomposition step in Algorithm 1 has a direct analogue in terms of a polynomial reduction in step 5 of Algorithm 2 for a suitable monomial ordering. Multiplying Eq. (B.1) by $q_{1}^{\alpha_{1}} \cdots q_{m}^{\alpha_{1}}$ and replacing $q_{i} d_{i}\left(x_{1}, \ldots\right)=1$ gives

$$
q_{1}^{\alpha_{1}} \cdots q_{m}^{\alpha_{m}}-\sum_{i} h_{i}\left(x_{1}, \ldots\right) q_{1}^{\alpha_{1}} \cdots \hat{q}_{i}^{\alpha_{i}} \cdots q_{m}^{\alpha_{m}}=0
$$

where $\hat{q}_{i}{ }^{\alpha_{i}}$ means leaving $q_{i}^{\alpha_{i}}$ out in the product. It is clear that the polynomial on the lhs of Eq. (B.3) is a member of the ideal $I=\left\langle 1-q_{1} d_{1}\left(x_{1}, \ldots\right), \ldots\right\rangle$. Furthermore, $q_{1}^{\alpha_{1}} \cdots q_{m}^{\alpha_{m}}$ is the leading monomial for any monomial ordering which sorts first for all of the $q_{1}, \ldots, q_{m}$ and then for the $x_{1}, \ldots, x_{n}$. In Algorithm 2 , that leading monomial will be thus be replaced by the subleading terms on the lhs of Eq. (B.3), resulting in terms with fewer factors of $q_{i}$, that is, simpler denominators. A fully reduced term will therefore not have all factors $q_{1} \cdots q_{n}$. This proves the above statements We conclude that, for our choice of monomial ordering, the polynomial reduction in Algorithm 2 guarantees requirement (i) 
of Leinnartas' decomposition, that is, the separation of independent denominator zeros.

Second, we consider requirement (ii), that is, the algebraic independence of the denominator factors. Let us assume that our decomposition contains a term with denominator $d_{1}^{\alpha_{1}} \cdots d_{m}^{\alpha_{m}}$, where the factors $\left\{d_{1}, \ldots d_{m}\right\}$ are algebraically dependent. One can show that then also the polynomials $\left\{d_{1}^{\alpha_{1}}, \ldots d_{m}^{\alpha_{m}}\right\}$ are algebraically dependent and there exists a non-zero annihilating polynomial $p\left(y_{1}, \ldots, y_{m}\right)$ with

$$
p\left(d_{1}^{\alpha_{1}}, \ldots, d_{m}^{\alpha_{m}}\right)=0
$$

after inserting the explicit expressions the $d_{i}\left(x_{1}, \ldots\right)$. In Algorithm 1, one can for example choose one of the monomials of $p$ with smallest degree and substitute the remainder, i.e. one has

$$
c_{\beta}\left(d^{\alpha}\right)^{\beta}=-\sum_{\gamma \in S} c_{\gamma}\left(d^{\alpha}\right)^{\gamma},
$$

where $d=\left(d_{1}, \ldots d_{m}\right), \alpha, \beta$ and $\gamma$ are multi-indices in $\mathbb{N}^{m}$, with $\sum_{i} \beta_{i} \leq \sum_{i} \gamma_{i}$ for all $\beta$ in the set of multi-indices $S$. Dividing by $c_{\beta} d^{\beta+1}$ gives the decomposition step [6]

$$
\frac{1}{d_{1}^{\alpha_{1}} \cdots d_{m}^{\alpha_{m}}}=-\sum_{\gamma \in S} \frac{c_{\gamma}}{c_{\beta}} \prod_{i=1}^{m} \frac{d_{i}^{\alpha_{i} \gamma_{i}}}{d_{i}^{\alpha_{i}\left(\beta_{i}+1\right)}} .
$$

Since $\sum_{i} \beta_{i} \leq \sum_{i} \gamma_{i}$ and $\beta \neq \gamma$, for each $\gamma$ there exists an $i$ such that $\beta_{i}+1 \leq \gamma_{i}$ and the factor $d_{i}^{\beta_{i}+1}$ is removed from the denominator. Therefore, each term on the rhs of (B.6) depends on at least one denominator factor $d_{i}$ less than the lhs.

The described decomposition step in Algorithm 1 may or may not have an analogue in terms of a polynomial reduction in step 5 of Algorithm 2, depending on the monomial ordering. In general, for a degree based ordering of the $q_{i}$ variables, either globally or in blocks like proposed in this paper, a similar decomposition will not occur. In (B.6), the denominator degree may actually be higher on the rhs than on the lhs, and it may therefore not correspond to a polynomial reduction for such an ordering. A lexicographic ordering, on the other hand, will separate algebraically dependent denominators. To show this, we pick in Eq. (B.4) the unique exponent $\beta^{\prime}$ of $p$ such that $\left(q^{\alpha}\right)^{\beta^{\prime}}$ is minimal with respect to our monomial ordering. Multiplication with $\left(q^{\alpha}\right)^{\beta^{\prime}+1} /\left(c_{\beta^{\prime}}\right)$ gives

$$
q_{1}^{\alpha_{1}} \cdots q_{m}^{\alpha_{m}}+\sum_{\gamma \in S} \frac{c_{\gamma}}{c_{\beta^{\prime}}} \prod_{i=1}^{m} d_{i}^{\alpha_{i} \gamma_{i}} q_{i}^{\alpha_{i}\left(\beta_{i}^{\prime}+1\right)}=0 .
$$

Replacing all products $q_{i} d_{i}\left(x_{1}, \ldots\right)=1$ gives

$$
q_{1}^{\alpha_{1}} \cdots q_{m}^{\alpha_{m}}+\sum_{\gamma \in S} \frac{c_{\gamma}}{c_{\beta^{\prime}}} \prod_{i=1}^{m} d_{i}^{\max \left(\alpha_{i}\left(\gamma_{i}-\beta_{i}^{\prime}-1\right), 0\right)} q_{i}^{\max \left(\alpha_{i}\left(\beta_{i}^{\prime}+1-\gamma_{i}\right), 0\right)}=0 .
$$


Assuming a lexicographic ordering of the $q_{i}$ with $q_{1} \succ q_{2} \succ \ldots$ implies that for each term $\gamma$ there exists a $j=1, \ldots, m$ such that $\beta_{i}^{\prime}=\gamma_{i}$ for all $i=1, \ldots, j$, $\beta_{j}^{\prime} \prec \gamma_{j}$, the powers of $q_{i}$ coincide with that of the first term for $i=1, \ldots, j-1$, but $q_{j}$ is removed. This means that the first term in (B.8) is indeed the leading term. Since furthermore the polynomial on the lhs of (B.8) is a member of the ideal $I=\left\langle q_{1} d_{1}\left(x_{1}, \ldots\right)-1, \ldots\right\rangle$, the first term in (B.8) would be reduced in step 5 of Algorithm 2 for this ordering. We conclude that, for the block monomial ordering presented in this article, the polynomial reduction in Algorithm 2 does not guarantee requirement (ii) of Leinnartas' decomposition, that is, algebraically independent denominators. In contrast, a lexicographical ordering guarantees this requirement.

We would like to illustrate the impact of the monomial ordering on the decomposition with the following example. We consider the irreducible denominator factors

$$
d_{1}=x^{3}+y^{4}, \quad d_{2}=x+y^{2}, \quad d_{3}=x^{2}+y,
$$

which share a common zero. We note that three denominator factors in two variables must be algebraically dependent on general grounds. Let us consider the ideal

$$
I=\left\langle q_{1} d_{1}(x, y)-1, q_{2} d_{2}(x, y)-1, q_{3} d_{3}(x, y)-1\right\rangle .
$$

The monomial block ordering proposed in this article gives

$$
\left\{\left\{q_{1}, q_{2}, q_{3}\right\},\{x, y\}\right\} .
$$

Calculating the Gröbner basis of $I$ with respect to this ordering, we see that the polynomial representation of $1 /\left(d_{1} d_{2} d_{3}\right)$,

$$
q_{1} q_{2} q_{3},
$$

is fully reduced already, despite the denominators $d_{1}, d_{2}$ and $d_{3}$ being algebraically dependent.

Next, let us consider a lexicographic ordering of the $q_{i}$ according to

$$
\left\{\left\{q_{1}\right\},\left\{q_{2}\right\},\left\{q_{3}\right\},\{x, y\}\right\} .
$$

In this case, the Gröbner basis computation of $I$ reveals that $q_{1} q_{2} q_{3}$ is reducible. Alternatively, we can derive a reduction identity of type (B.8) as described above. Indeed, it is not difficult to calculate the annihilator

$$
\begin{aligned}
p & \left(y_{1}, y_{2}, y_{3}\right)=y_{1}^{4}-4 y_{1}^{3} y_{2}^{2}-4 y_{1}^{3} y_{3}+3 y_{1}^{3}+6 y_{1}^{2} y_{2}^{4}+4 y_{1}^{2} y_{2}^{2} y_{3}-y_{1}^{2} y_{2}^{2}+8 y_{1}^{2} y_{2} y_{3}^{2} \\
& -6 y_{1}^{2} y_{2} y_{3}-8 y_{1}^{2} y_{2}-2 y_{1}^{2} y_{3}^{3}+6 y_{1}^{2} y_{3}^{2}-2 y_{1}^{2} y_{3}+3 y_{1}^{2}-4 y_{1} y_{2}^{6}+4 y_{1} y_{2}^{4} y_{3} \\
& -7 y_{1} y_{2}^{4}-16 y_{1} y_{2}^{3} y_{3}^{2}+12 y_{1} y_{2}^{3} y_{3}-8 y_{1} y_{2}^{3}+4 y_{1} y_{2}^{2} y_{3}^{3}+4 y_{1} y_{2}^{2} y_{3}^{2}+36 y_{1} y_{2}^{2} y_{3} \\
& -16 y_{1} y_{2} y_{3}^{3}-18 y_{1} y_{2} y_{3}^{2}+2 y_{1} y_{2} y_{3}-5 y_{1} y_{2}+4 y_{1} y_{3}^{4}+2 y_{1} y_{3}^{3}-y_{1} y_{3}^{2}+2 y_{1} y_{3} \\
& +y_{2}^{8}-4 y_{2}^{6} y_{3}+5 y_{2}^{6}+8 y_{2}^{5} y_{3}^{2}-6 y_{2}^{5} y_{3}-2 y_{2}^{4} y_{3}^{3}+6 y_{2}^{4} y_{3}^{2}-2 y_{2}^{4} y_{3}+5 y_{2}^{4} \\
& -16 y_{2}^{3} y_{3}^{3}-14 y_{2}^{3} y_{3}^{2}-2 y_{2}^{3} y_{3}+20 y_{2}^{2} y_{3}^{4}+6 y_{2}^{2} y_{3}^{3}-8 y_{2} y_{3}^{5}+5 y_{2} y_{3}^{4}+y_{3}^{6}-2 y_{3}^{5}
\end{aligned}
$$


with $p\left(d_{1}(x, y), d_{2}(x, y), d_{3}(x, y)\right)=0$ and identify $q_{i} d_{i}=1$ modulo $I$ to obtain

$$
\begin{aligned}
& q_{1} q_{2} q_{3}-\frac{1}{2} q_{1} q_{2}-\frac{1}{2}\left(d_{2}^{7}+5 d_{2}^{5}+5 d_{2}^{3}\right) q_{1} q_{3}^{6}+\left(2 d_{2}^{5}+3 d_{2}^{4}+d_{2}^{3}+d_{2}^{2}\right) q_{1} q_{3}^{5} \\
& -\left(4 d_{2}^{4}+3 d_{2}^{3}-7 d_{2}^{2}\right) q_{1} q_{3}^{4}+\left(d_{2}^{3}+8 d_{2}^{2}-3 d_{2}\right) q_{1} q_{3}^{3}-\frac{1}{2}\left(20 d_{2}+5\right) q_{1} q_{3}^{2}+4 q_{1} q_{3} \\
& -\frac{1}{2}\left(d_{1}^{3}+3 d_{1}^{2}+3 d_{1}\right) q_{2} q_{3}^{6}+\left(2 d_{1}^{2}+d_{1}-1\right) q_{2} q_{3}^{5}-\frac{1}{2}\left(6 d_{1}-1\right) q_{2} q_{3}^{4}+\left(d_{1}-1\right) q_{2} q_{3}^{3} \\
& -2 q_{2} q_{3}^{2}+\frac{1}{2}\left(4 d_{1}^{2} d_{2}-6 d_{1} d_{2}^{3}+d_{1} d_{2}+8 d_{1}+4 d_{2}^{5}+7 d_{2}^{3}+8 d_{2}^{2}+5\right) q_{3}^{6} \\
& -\left(2 d_{1} d_{2}-3 d_{1}+2 d_{2}^{3}+6 d_{2}^{2}+18 d_{2}+1\right) q_{3}^{5}-\left(4 d_{1}-8 d_{2}^{2}+2 d_{2}-9\right) q_{3}^{4} \\
& -\left(2 d_{2}-8\right) q_{3}^{3}=0,
\end{aligned}
$$

where $d_{1}, d_{2}, d_{3}$ are meant to be replaced by their definitions (B.9). As we see, this gives a reduction identity for (B.12) since the first term is indeed the leading term in this ordering, such that the algebraically dependent denominators are

decomposed. However, we also see that this decomposition leads to a significant increase in the degrees of the polynomials.

\section{References}

[1] H. Strubbe, Manual for Schoonschip: A CDC 6000 / 7000 program for symbolic evaluation of algebraic expressions (program made by $M$. Veltman), Comput. Phys. Commun. 8 (1974) 1.

[2] E. Remiddi, "Computer algebra, past, present and future." Talk given at Tini 80 Fest, 2011, https://www.nikhef.nl/ t45/Tini80Fest/Remiddi.pdf

[3] J. A. M. Vermaseren, New features of FORM, math-ph/0010025

[4] B. Ruijl, T. Ueda and J. Vermaseren, FORM version 4.2, 1707.06453 ,

[5] E. K. LeĬnartas, Factorization of rational functions of several variables into partial fractions, Soviet Math. (Iz. VUZ) 22 (1978) 35.

[6] A. Raichev, Leĭnartas' partial fraction decomposition, 1206.4740.

[7] C. Meyer, Transforming differential equations of multi-loop Feynman integrals into canonical form, JHEP 04 (2017) 006 [1611.01087.

[8] C. Meyer, Algorithmic transformation of multi-loop master integrals to a canonical basis with CANONICA, Comput. Phys. Commun. 222 (2018) 295 [1705.06252].

[9] S. Abreu, J. Dormans, F. Febres Cordero, H. Ita, B. Page and V. Sotnikov, Analytic Form of the Planar Two-Loop Five-Parton Scattering Amplitudes in QCD, JHEP 05 (2019) 084 [1904.00945.

[10] D. A. Cox, J. Little and D. O'Shea, Ideals, Varieties, and Algorithms. Springer, Cham, 2015, 10.1007/978-3-319-16721-3. 
[11] M. Kreuzer and L. Robbiano, Computational commutative algebra. 1. Springer, Berlin, Heidelberg, 2000, 10.1007/978-3-540-70628-1.

[12] J. Boehm, M. Wittmann, Z. Wu, Y. Xu and Y. Zhang, IBP reduction coefficients made simple, JHEP 12 (2020) 054 [2008.13194].

[13] A. von Manteuffel and R. M. Schabinger, A novel approach to integration by parts reduction, Phys. Lett. B744 (2015) 101 [1406.4513].

[14] A. von Manteuffel and R. M. Schabinger, Quark and gluon form factors to four-loop order in QCD: the $N_{f}^{3}$ contributions, Phys. Rev. D 95 (2017) 034030 [1611.00795].

[15] T. Peraro, Scattering amplitudes over finite fields and multivariate functional reconstruction, JHEP 12 (2016) 030 [1608.01902].

[16] T. Peraro, FiniteFlow: multivariate functional reconstruction using finite fields and dataflow graphs, JHEP 07 (2019) 031 [1905.08019].

[17] J. Klappert and F. Lange, Reconstructing rational functions with FireFly, Comput. Phys. Commun. 247 (2020) 106951 [1904.00009].

[18] A. Smirnov and F. Chuharev, FIRE6: Feynman Integral REduction with Modular Arithmetic, 1901.07808

[19] A. Smirnov and V. Smirnov, How to choose master integrals, Nucl. Phys. B 960 (2020) 115213 [2002.08042].

[20] J. Usovitsch, Factorization of denominators in integration-by-parts reductions, 2002.08173.

[21] M. Heller, O. Tomalak, M. Vanderhaeghen and S. Wu, Leading Order Corrections to the Bethe-Heitler Process in the $\gamma p \rightarrow l^{+} l^{-} p$ Reaction, Phys. Rev. D 100 (2019) 076013 [1906.02706].

[22] M. Heller, N. Keil and M. Vanderhaeghen, Leading-order QED radiative corrections to timelike Compton scattering on the proton, 2012.09565

[23] R. Tarrach, Invariant Amplitudes for Virtual Compton Scattering Off Polarized Nucleons Free from Kinematical Singularities, Zeros and Constraints, Nuovo Cim. A 28 (1975) 409.

[24] B. Agarwal, S. P. Jones and A. von Manteuffel, Two-loop helicity amplitudes for $g g \rightarrow Z Z$ with full top-quark mass effects, 2011.15113.

[25] D. Bendle, J. Böhm, W. Decker, A. Georgoudis, F.-J. Pfreundt, M. Rahn et al., Integration-by-parts reductions of Feynman integrals using Singular and GPI-Space, JHEP 02 (2020) 079 [1908.04301].

[26] F. Brown, The Massless higher-loop two-point function, Commun. Math. Phys. 287 (2009) 925 [0804.1660]. 
[27] E. Panzer, Algorithms for the symbolic integration of hyperlogarithms with applications to Feynman integrals, Comput. Phys. Commun. 188 (2015) 148 [1403.3385]. 